\title{
Article \\ Applications of a Multiplier Transformation and Ruscheweyh Derivative for Obtaining New Strong Differential Subordinations
}

\author{
Alina Alb Lupaş (D) \\ Department of Mathematics and Computer Science, University of Oradea, 1 Universitatii Street, \\ 410087 Oradea, Romania; dalb@uoradea.ro or alblupas@gmail.com
}

check for updates

Citation: Lupaş, A.A. Applications of a Multiplier Transformation and Ruscheweyh Derivative for Obtaining New Strong Differential Subordinations. Symmetry 2021, 13, 1312. https://doi.org/10.3390/ sym13081312

Academic Editor: Ioan Rașa

Received: 11 July 2021

Accepted: 19 July 2021

Published: 21 July 2021

Publisher's Note: MDPI stays neutral with regard to jurisdictional claims in published maps and institutional affiliations.

Copyright: (C) 2021 by the author. Licensee MDPI, Basel, Switzerland. This article is an open access article distributed under the terms and conditions of the Creative Commons Attribution (CC BY) license (https:/ / creativecommons.org/licenses/by/ $4.0 /)$.
Abstract: Here, we study strong differential subordinations for the extended new operator $I R_{\lambda, l}^{m}$ defined by the Hadamard product of the extended multiplier transformation $I(m, \lambda, l)$ and the extended Ruscheweyh derivative $R^{m}$, on the class of normalized analytic functions $\mathcal{A}_{n \zeta}^{*}=\{f \in$ $\left.\mathcal{H}(U \times \bar{U}), f(z, \zeta)=z+a_{n+1}(\zeta) z^{n+1}+\ldots, z \in U, \zeta \in \bar{U}\right\}$, by $I R_{\lambda, l}^{m}: \mathcal{A}_{n \zeta}^{*} \rightarrow \mathcal{A}_{n \zeta}^{*}, I R_{\lambda, l}^{m} f(z, \zeta)=$ $\left(I(m, \lambda, l) * R^{m}\right) f(z, \zeta)$.

Keywords: strong differential subordination; univalent function; convex function; best dominant; extended differential operator; convolution product

\section{Introduction}

Different types of operators have been used from early on in the study of complex functions. Among the advantages of using operators is the possibility of giving easier proofs of already known results but also facilitating the emergence of new, original research. The best-known operators are integral and differential operators. The first integral operator was introduced in 1915 [1] by Alexander. A very well-known integral operator was introduced by Libera in 1965; Bernardi then generalized it in 1969 [2]. Some of the best-known differential operators are the one used for obtaining the original results of the present paper, introduced by Ruscheweyh in 1975 [3], and Sălăgean's differential operator, introduced in 1983 [4]. Recent studies have been conducted combining these two kinds of operators, obtaining differential-integral operators, such as seen in [5-7]. Convolution operators having numerous applications have been introduced, such as the Dziok-Srivastava operator [8], Srivastava-Wright operator [9], the operator introduced in [10] and the operator used in the present paper, defined in [11]. The monograph [12] and the paper [13], as well as the research performed in [14], give hints as to how the differential operator method is linked to partial differential equations and their applications.

For the research presented in the present paper, the following notions are necessary.

Consider $U=\{z \in \mathbb{C}:|z|<1\}$ the unit disc of the complex plane, the closed unit disc of the complex plane $\bar{U}=\{z \in \mathbb{C}:|z| \leq 1\}$ and the class of analytic functions in $U \times \bar{U}$ denoted $\mathcal{H}(U \times \bar{U})$.

Let

$$
\mathcal{A}_{n \zeta}^{*}=\left\{f \in \mathcal{H}(U \times \bar{U}): f(z, \zeta)=z+a_{n+1}(\zeta) z^{n+1}+\ldots, z \in U, \zeta \in \bar{U}\right\},
$$

where $a_{k}(\zeta)$ are holomorphic functions in $\bar{U}$ for $k \geq 2$, and

$\mathcal{H}^{*}[a, n, \zeta]=\left\{f \in \mathcal{H}(U \times \bar{U}): f(z, \zeta)=a+a_{n}(\zeta) z^{n}+a_{n+1}(\zeta) z^{n+1}+\ldots, z \in U, \zeta \in \bar{U}\right\}$, for $a \in \mathbb{C}$ and $n \in \mathbb{N}, a_{k}(\zeta)$ are holomorphic functions in $\bar{U}$ for $k \geq n$.

J.A. Antonino and S. Romaguera defined in [15] the notion of strong differential subordinations, which was developed by G.I. Oros and Gh. Oros in [16,17]. 
Definition 1. ([16]) Let $f(z, \zeta), H(z, \zeta)$ be analytic in $U \times \bar{U}$. The function $f(z, \zeta)$ is said to be strongly subordinate to $H(z, \zeta)$ if there exists a function $w$ analytic in $U$, with $w(0)=0$ and $|w(z)|<1$, such that $f(z, \zeta)=H(w(z), \zeta)$ for all $\zeta \in \bar{U}$. In such a case, we write $f(z, \zeta) \prec \prec H(z, \zeta), z \in U, \zeta \in \bar{U}$.

Remark 1. ([16]) (i) Since $f(z, \zeta)$ is analytic in $U \times \bar{U}$, for all $\zeta \in \bar{U}$, and univalent in $U$, for all $\zeta \in \bar{U}$, Definition 1 is equivalent to $f(0, \zeta)=H(0, \zeta)$, for all $\zeta \in \bar{U}$, and $f(U \times \bar{U}) \subset$ $H(U \times \bar{U})$.

(ii) If $H(z, \zeta) \equiv H(z)$ and $f(z, \zeta) \equiv f(z)$, the strong subordination becomes the usual notion of subordination.

The concept of differential subordination was introduced in $[18,19]$ by S.S. Miller and P.T. Mocanu. The main results related to the theory of differential subordination can be found in [20] and we next recall some basic definitions as given in this monograph.

Definition 2. ([20]) Let $f, F \in \mathcal{H}(U)$. The function $f$ is said to be subordinate to $F$, if there exists a Schwarz function $w$, analytic in $U$, with $w(0)=0$ and $|w(z)|<1, z \in U$, such that $f(z)=F(w(z)), z \in U$. In such a case, we write $f \prec F$. If $F$ is univalent, then $f \prec F$ if and only if $f(0)=g(0)$ and $f(U) \subset g(U)$.

Definition 3. ([20]) Let $\psi: \mathbb{C}^{3} \times U \rightarrow \mathbb{C}$ and let $h$ be univalent in $U$. If $p$ is analytic and satisfies the differential subordination

$$
\psi\left(p(z), z p^{\prime}(z), z^{2} p^{\prime \prime}(z) ; z\right) \prec h(z), z \in U,
$$

then $p$ is called a solution of the differential subordination. The univalent function $q$ is called $a$ dominant of the solutions of the differential subordination, or, more simply, a dominant, if $p \prec q$ for all $p$ satisfying (1). A dominant $\widetilde{q}$ that satisfies $\widetilde{q} \prec q$ for all dominants $q$ of (1) is said to be the best dominant of (1).

In studying the strong differential subordinations, we will use the following lemmas.

Lemma 1. ([21]) Let $h(z, \zeta)$ be a convex function with $h(0, \zeta)=$ a for every $\zeta \in \bar{U}$ and let $\gamma \in \mathbb{C}^{*}$ be a complex number with Rer $\geq 0$. If $p \in \mathcal{H}^{*}[a, n, \zeta]$ and

$$
p(z, \zeta)+\frac{1}{\gamma} z p_{z}^{\prime}(z, \zeta) \prec \prec h(z, \zeta)
$$

then

$$
p(z, \zeta) \prec \prec g(z, \zeta) \prec \prec h(z, \zeta),
$$

where $g(z, \zeta)=\frac{\gamma}{n z^{\frac{\gamma}{n}}} \int_{0}^{z} h(t, \zeta) t^{\frac{\gamma}{n}-1} d t$ is convex and it is the best dominant.

Lemma 2. ([21]) Let $g(z, \zeta)$ be a convex function in $U \times \bar{U}$, for all $\zeta \in \bar{U}$, and let

$$
h(z, \zeta)=g(z, \zeta)+n \alpha z g_{z}^{\prime}(z, \zeta), \quad z \in U, \zeta \in \bar{U},
$$

where $\alpha>0$ and $n$ is a positive integer. If

$$
p(z, \zeta)=g(0, \zeta)+p_{n}(\zeta) z^{n}+p_{n+1}(\zeta) z^{n+1}+\ldots, \quad z \in U, \zeta \in \bar{U},
$$

is holomorphic in $U \times \bar{U}$ and

$$
p(z, \zeta)+\alpha z p_{z}^{\prime}(z, \zeta) \prec \prec h(z, \zeta), \quad z \in U, \zeta \in \bar{U},
$$

then

$$
p(z, \zeta) \prec \prec g(z, \zeta)
$$


and this result is sharp.

The author extended in [22,23] the multiplier transformation ([24]) and, respectively, the Ruscheweyh derivative ([3]) to the new class of analytic functions $\mathcal{A}_{n \zeta}^{*}$ introduced in [17].

Definition 4. ([22]) For $f \in \mathcal{A}_{n \zeta^{\prime}}^{*}, m \in \mathbb{N} \cup\{0\}, \lambda, l \geq 0$, the operator $I(m, \lambda, l): \mathcal{A}_{n \zeta}^{*} \rightarrow \mathcal{A}_{n \zeta}^{*}$ is defined by the following infinite series:

$$
I(m, \lambda, l) f(z, \zeta):=z+\sum_{j=n+1}^{\infty}\left(\frac{1+\lambda(j-1)+l}{l+1}\right)^{m} a_{j}(\zeta) z^{j} .
$$

Remark 2. ([22]) The operator $I(m, \lambda, l)$ verifies the property

$$
(l+1) I(m+1, \lambda, l) f(z, \zeta)=[l+1-\lambda] I(m, \lambda, l) f(z, \zeta)+\lambda z(I(m, \lambda, l) f(z, \zeta))^{\prime},
$$

$\zeta \in \bar{U}, z \in U$

Definition 5. ([23]) For $f \in \mathcal{A}_{n \zeta}^{*}, m \in \mathbb{N}$, the operator $R^{m}$ is defined by $R^{m}: \mathcal{A}_{n \zeta}^{*} \rightarrow \mathcal{A}_{n \zeta^{\prime}}^{*}$

$$
\begin{aligned}
R^{0} f(z, \zeta)= & f(z, \zeta) \\
R^{1} f(z, \zeta)= & z f^{\prime}(z, \zeta) \\
& \cdots \\
(m+1) R^{m+1} f(z, \zeta)= & z\left(R^{m} f(z, \zeta)\right)^{\prime}+m R^{m} f(z, \zeta), \quad \zeta \in \bar{U}, z \in U .
\end{aligned}
$$

Remark 3. ([23]) If $f \in \mathcal{A}_{n \zeta}^{*}, f(z, \zeta)=z+\sum_{j=n+1}^{\infty} a_{j}(\zeta) z^{j}$, then $R^{m} f(z, \zeta)=z+\sum_{j=n+1}^{\infty} C_{m+j-1}^{m} a_{j}(\zeta) z^{j}, \zeta \in \bar{U}, z \in U$.

The author also extended in [11] the differential operator obtained as a convolution product (Hadamard product) of multiplier transformation and the Ruscheweyh derivative $([25,26])$ to the class $\mathcal{A}_{n \zeta}^{*}$.

Definition 6. ([11]) Let $\lambda, l \geq 0$ and $m \in \mathbb{N}$. Denote by $I R_{\lambda, l}^{m}$ the extended operator given by the Hadamard product of the extended multiplier transformation $I(m, \lambda, l)$ and the extended Ruscheweyh derivative $R^{m}, I R_{\lambda, l}^{m}: \mathcal{A}_{n \zeta}^{*} \rightarrow \mathcal{A}_{n \zeta^{\prime}}^{*}$

$$
I R_{\lambda, l}^{m} f(z, \zeta)=\left(I(m, \lambda, l) * R^{m}\right) f(z, \zeta)
$$

Remark 4. ([11]) If $f \in \mathcal{A}_{n \zeta^{\prime}}^{*} f(z, \zeta)=z+\sum_{j=n+1}^{\infty} a_{j}(\zeta) z^{j}$, then

$$
I R_{\lambda, l}^{m} f(z, \zeta)=z+\sum_{j=n+1}^{\infty}\left(\frac{1+\lambda(j-1)+l}{l+1}\right)^{m} C_{m+j-1}^{m} a_{j}^{2}(\zeta) z^{j}, \zeta \in \bar{U}, z \in U .
$$

Remark 5. For $l=0, \lambda \geq 0$, we obtain the operator $D R_{\lambda}^{n}$ studied in [27], and for $l=0$ and $\lambda=1$, we obtain the operator $S R^{n}$ studied in [28].

The symmetry properties of the functions used in defining an equation or inequality could be studied to determine solutions with particular properties. Regarding the differential subordinations or strong differential subordinations, which are some inequalities, the study of special functions, given their symmetry properties, could provide interesting results. Studies on the symmetry properties for different types of operators associated with the concept of quantum computing could also be investigated in a future paper. 


\section{Main Results}

Definition 7. Consider $m \in \mathbb{N}, \lambda, l \geq 0$ and $\delta \in[0,1)$. A function $f \in \mathcal{A}_{n \zeta}^{*}$ belongs to the class $\mathcal{I R}_{m}(\delta, \lambda, l, \zeta)$ if the inequality

$$
\operatorname{Re}\left(I R_{\lambda, l}^{m} f(z, \zeta)\right)_{z}^{\prime}>\delta, \quad \zeta \in \bar{U}, z \in U,
$$

is satisfied.

Theorem 1. Let $g$ be a convex function in $U \times \bar{U}$ such that $g(0, \zeta)=1$ and $h(z, \zeta)=g(z, \zeta)+$ $\frac{1}{c+2} z g_{z}^{\prime}(z, \zeta)$, with $c>0$ and $\zeta \in \bar{U}, z \in U$. If $n, m \in \mathbb{N}, \lambda, l \geq 0, f \in \mathcal{I R}_{m}(\delta, \lambda, l, \zeta)$ and $F(z, \zeta)=I_{c}(f)(z, \zeta)=\frac{c+2}{z^{c+1}} \int_{0}^{z} t^{c} f(t, \zeta) d t, \zeta \in \bar{U}, z \in U$, then

$$
\left(I R_{\lambda, l}^{m} f(z, \zeta)\right)_{z}^{\prime} \prec \prec h(z, \zeta), \zeta \in \bar{U}, z \in U,
$$

implies

$$
\left(I R_{\lambda, l}^{m} F(z, \zeta)\right)_{z}^{\prime} \prec \prec g(z, \zeta), \zeta \in \bar{U}, z \in U,
$$

and this result is sharp.

Proof. We have

$$
z^{c+1} F(z, \zeta)=(c+2) \int_{0}^{z} t^{c} f(t, \zeta) d t
$$

and differentiating it with respect to $z$, we obtain $(c+1) F(z, \zeta)+z F_{z}^{\prime}(z, \zeta)=(c+2) f(z, \zeta)$, which implies the relation

$$
(c+1) I R_{\lambda, l}^{m} F(z, \zeta)+z\left(I R_{\lambda, l}^{m} F(z, \zeta)\right)_{z}^{\prime}=(c+2) I R_{\lambda, l}^{m} f(z, \zeta), \quad \zeta \in \bar{U}, z \in U,
$$

which, by differentiating with respect to $z$, gives

$$
\left(I R_{\lambda, l}^{m} F(z, \zeta)\right)_{z}^{\prime}+\frac{1}{c+2} z\left(I R_{\lambda, l}^{m} F(z, \zeta)\right)_{z^{2}}^{\prime \prime}=\left(I R_{\lambda, l}^{m} f(z, \zeta)\right)_{z^{\prime}}^{\prime} \quad \zeta \in \bar{U}, z \in U .
$$

Therefore, the strong differential subordination (3) becomes

$$
\left(I R_{\lambda, l}^{m} F(z, \zeta)\right)_{z}^{\prime}+\frac{1}{c+2} z\left(I R_{\lambda, l}^{m} F(z, \zeta)\right)_{z^{2}}^{\prime \prime} \prec \prec g(z, \zeta)+\frac{1}{c+2} z g_{z}^{\prime}(z, \zeta) .
$$

Denote

$$
p(z, \zeta)=\left(I R_{\lambda, l}^{m} F(z, \zeta)\right)_{z^{\prime}}^{\prime} \quad \zeta \in \bar{U}, z \in U .
$$

Replacing (8) in (7), we obtain

$$
p(z, \zeta)+\frac{1}{c+2} z p_{z}^{\prime}(z, \zeta) \prec \prec g(z, \zeta)+\frac{1}{c+2} z g_{z}^{\prime}(z, \zeta), \quad \zeta \in \bar{U}, z \in U .
$$

Applying Lemma 2, we obtain the sharp result

$$
p(z, \zeta) \prec \prec g(z, \zeta), \quad \zeta \in \bar{U}, z \in U,
$$

equivalently with

$$
\left(I R_{\lambda, l}^{m} F(z, \zeta)\right)_{z}^{\prime} \prec \prec g(z, \zeta), \zeta \in \bar{U}, z \in U .
$$


Theorem 2. Let $\delta \in[0,1)$ and $h(z, \zeta)=\frac{\zeta+(2 \delta-\zeta) z}{1+z}, z \in U, \zeta \in \bar{U}$. If $m \in \mathbb{N}, \lambda, l \geq 0, c>0$ and $I_{\mathcal{C}}$ is given by Theorem 1 , then

$$
I_{c}\left[\mathcal{I R}_{m}(\delta, \lambda, l, \zeta)\right] \subset \mathcal{I R}_{m}\left(\delta^{*}, \lambda, l, \zeta\right),
$$

with $\delta^{*}=2 \delta-\zeta+\frac{2(c+2)(\zeta-\delta)}{n} \beta\left(\frac{c+2}{n}-2\right)$ and $\beta(x)=\int_{0}^{1} \frac{t^{x+1}}{t+1} d t$.

Proof. The same steps as in the proof of Theorem 1 for the convex function $h$ give

$$
p(z, \zeta)+\frac{1}{c+2} z p_{z}^{\prime}(z, \zeta) \prec \prec h(z, \zeta),
$$

with $p(z, \zeta)$ defined in (8).

For $\gamma=c+2$, Lemma 1 gives

$$
p(z, \zeta) \prec \prec g(z, \zeta) \prec \prec h(z, \zeta),
$$

equivalently with

$$
\left(I R_{\lambda, l}^{m} F(z, \zeta)\right)_{z}^{\prime} \prec \prec g(z, \zeta) \prec \prec h(z, \zeta),
$$

and

$$
\begin{aligned}
g(z, \zeta)= & \frac{c+2}{n z^{\frac{c+2}{n}}} \int_{0}^{z} t^{\frac{c+2}{n}-1} \frac{\zeta+(2 \delta-\zeta) t}{1+t} d t= \\
& (2 \delta-\zeta)+\frac{2(c+2)(\zeta-\delta)}{n z^{\frac{c+2}{n}}} \int_{0}^{z} \frac{t^{\frac{c+2}{n}-1}}{1+t} d t
\end{aligned}
$$

$g$ being convex and $g(U \times \bar{U})$ being symmetric with respect to the real axis, we obtain

$$
\begin{gathered}
\operatorname{Re}\left(I R_{\lambda, l}^{m} F(z, \zeta)\right)_{z}^{\prime} \geq \min _{|z|=1} \operatorname{Re} g(z, \zeta)=\operatorname{Re} g(1, \zeta)=\delta^{*}= \\
2 \delta-\zeta+\frac{2(c+2)(\zeta-\delta)}{n} \beta\left(\frac{c+2}{n}-2\right) .
\end{gathered}
$$

Theorem 3. Let $g$ be a convex function in $U \times \bar{U}, g(0, \zeta)=1$ and $h(z, \zeta)=g(z, \zeta)+z g_{z}^{\prime}(z, \zeta)$, $z \in U, \zeta \in \bar{U}$. If $m \in \mathbb{N}, \lambda, l \geq 0, f \in \mathcal{A}_{n \zeta}^{*}$ and

$$
\left(I R_{\lambda, l}^{m} f(z, \zeta)\right)_{z}^{\prime} \prec \prec h(z, \zeta), \zeta \in \bar{U}, z \in U,
$$

the strong differential subordination holds; then,

$$
\frac{I R_{\lambda, l}^{m} f(z, \zeta)}{z} \prec \prec g(z, \zeta), \zeta \in \bar{U}, z \in U,
$$

and this result is sharp.

Proof. Consider $f(z, \zeta)=z+\sum_{j=n+1}^{\infty} a_{j}(\zeta) z^{j} \in \mathcal{A}_{n \zeta}^{*}$ and $I R_{\lambda, l}^{m} f(z, \zeta)=z+\sum_{j=n+1}^{\infty}\left(\frac{1+\lambda(j-1)+l}{l+1}\right)^{m} C_{m+j-1}^{m} a_{j}^{2}(\zeta) z^{j}, \zeta \in \bar{U}, z \in U$.
$\quad$ Put $p(z, \zeta)=\frac{I R_{\lambda, l}^{m} f(z, \zeta)}{z}=1+\sum_{j=n+1}^{\infty}\left(\frac{1+\lambda(j-1)+l}{l+1}\right)^{m} C_{m+j-1}^{m} a_{j}^{2}(\zeta) z^{j-1}$.

Differentiating it with respect to $z$, we can write $p(z, \zeta)+z p_{z}^{\prime}(z, \zeta)=\left(I R_{\lambda, l}^{m} f(z, \zeta)\right)_{z^{\prime}}^{\prime}$ $\zeta \in \bar{U}, z \in U$. 
Relation (11) becomes $p(z, \zeta)+z p_{z}^{\prime}(z, \zeta) \prec \prec h(z, \zeta)=g(z, \zeta)+z g_{z}^{\prime}(z, \zeta)$ and Lemma 2 allows us to write $p(z, \zeta) \prec \prec g(z, \zeta), \zeta \in \bar{U}, z \in U$, which is equivalent with $\frac{I R_{\lambda, l}^{m} f(z, \zeta)}{z} \prec \prec$ $g(z, \zeta), \zeta \in \bar{U}, z \in U$.

Theorem 4. Let $h$ be a convex function in $U \times \bar{U}$ with $h(0, \zeta)=1$. If $m, n \in \mathbb{N}, \lambda, l \geq 0$, $f \in \mathcal{A}_{n \zeta}^{*}$ and

$$
\left(I R_{\lambda, l}^{m} f(z, \zeta)\right)_{z}^{\prime} \prec \prec h(z, \zeta), \zeta \in \bar{U}, z \in U,
$$

the strong differential subordination holds; then,

$$
\frac{I R_{\lambda, l}^{m} f(z, \zeta)}{z} \prec \prec g(z, \zeta) \prec \prec h(z, \zeta), \zeta \in \bar{U}, z \in U,
$$

where the convex best dominant is $g(z, \zeta)=\frac{1}{n z^{\frac{1}{n}}} \int_{0}^{z} h(t, \zeta) t^{\frac{1}{n}-1} d t$

Proof. For $f(z, \zeta)=z+\sum_{j=n+1}^{\infty} a_{j}(\zeta) z^{j} \in \mathcal{A}_{n \zeta}^{*}$, we can write $I R_{\lambda, l}^{m} f(z, \zeta)=z+\sum_{j=n+1}^{\infty}\left(\frac{1+\lambda(j-1)+l}{l+1}\right)^{m} C_{m+j-1}^{m} a_{j}^{2}(\zeta) z^{j}, \zeta \in \bar{U}, z \in U$.

Define $p(z, \zeta)=\frac{I R_{\lambda, l}^{m} f(z, \zeta)}{z}=1+\sum_{j=n+1}^{\infty}\left(\frac{1+\lambda(j-1)+l}{l+1}\right)^{m} C_{m+j-1}^{m} a_{j}^{2}(\zeta) z^{j-1} \in \mathcal{H}^{*}[1, n, \zeta]$.

Differentiating it with respect to $z$, we obtain $p(z, \zeta)+z p_{z}^{\prime}(z, \zeta)=\left(\operatorname{IR}_{\lambda, l}^{m} f(z, \zeta)\right)_{z^{\prime}}^{\prime}$ $\zeta \in \bar{U}, z \in U$, and the strong differential subordination from the hypothesis becomes $p(z, \zeta)+z p_{z}^{\prime}(z, \zeta) \prec \prec h(z, \zeta), \zeta \in \bar{U}, z \in U$. Applying Lemma 1 for $\gamma=1$, we obtain $p(z, \zeta) \prec \prec g(z, \zeta) \prec \prec h(z, \zeta), \zeta \in \bar{U}, z \in U$, and $g(z, \zeta)$ is the best dominant and it is convex. It is equivalent with $\frac{I R_{\lambda, l}^{m} f(z, \zeta)}{z} \prec \prec g(z, \zeta)=\frac{1}{n z^{\frac{1}{n}}} \int_{0}^{z} h(t, \zeta) t^{\frac{1}{n}-1} d t \prec \prec h(z, \zeta)$, $\zeta \in \bar{U}, z \in U$.

Corollary 1. Consider $0 \leq \beta<1$ and the convex function in $U \times \bar{U}, h(z, \zeta)=\frac{\zeta+(2 \beta-\zeta) z}{1+z}$. If $m, n \in \mathbb{N}, \lambda, l \geq 0, f \in \mathcal{A}_{n \zeta}^{*}$ and

$$
\left(I R_{\lambda, l}^{m} f(z, \zeta)\right)_{z}^{\prime} \prec \prec h(z, \zeta), \quad \zeta \in \bar{U}, z \in U,
$$

the strong differential subordination holds; then,

$$
\frac{I R_{\lambda, l}^{m} f(z, \zeta)}{z} \prec \prec g(z, \zeta) \prec \prec h(z, \zeta), \zeta \in \bar{U}, z \in U,
$$

where $g$ is the convex best dominant in $U \times \bar{U}$ given by $g(z, \zeta)=2 \beta-\zeta+\frac{2(\zeta-\beta)}{n z^{\frac{1}{n}}} \int_{0}^{z} \frac{t^{\frac{1}{n}-1}}{1+t} d t$.

Proof. Considering $p(z, \zeta)=\frac{I R_{\lambda, l}^{m} f(z, \zeta)}{z}$, the strong differential subordination (13) becomes

$$
p(z, \zeta)+z p_{z}^{\prime}(z, \zeta) \prec \prec h(z, \zeta)=\frac{\zeta+(2 \beta-\zeta) z}{1+z}, \quad \zeta \in \bar{U}, z \in U,
$$

and for $\gamma=1$, Lemma 1 implies $p(z, \zeta) \prec \prec g(z, \zeta) \prec \prec h(z, \zeta), z \in U, \zeta \in \bar{U}$, which is equivalent with

$$
\begin{aligned}
\frac{I R_{\lambda, l}^{m} f(z, \zeta)}{z} & \prec \prec g(z, \zeta)=\frac{1}{n z^{\frac{1}{n}}} \int_{0}^{z} h(t, \zeta) t^{\frac{1}{n}-1} d t=\frac{1}{n z^{\frac{1}{n}}} \int_{0}^{z} t^{\frac{1}{n}-1} \frac{\zeta+(2 \beta-\zeta) t}{1+t} d t \\
& =2 \beta-\zeta+\frac{2(\zeta-\beta)}{n z^{\frac{1}{n}}} \int_{0}^{z} \frac{t^{\frac{1}{n}-1}}{1+t} d t, \quad \zeta \in \bar{U}, z \in U .
\end{aligned}
$$


Theorem 5. Let $g$ be a convex function in $U \times \bar{U}$ such that $g(0, \zeta)=1$ and $h(z, \zeta)=g(z, \zeta)+$ $z g_{z}^{\prime}(z, \zeta), \zeta \in \bar{U}, z \in U$. If $m \in \mathbb{N}, \lambda, l \geq 0, f \in \mathcal{A}_{n \zeta}^{*}$ and

$$
\left(\frac{z I R_{\lambda, l}^{m+1} f(z, \zeta)}{I R_{\lambda, l}^{m} f(z, \zeta)}\right)_{z}^{\prime} \prec \prec h(z, \zeta), \quad \zeta \in \bar{U}, z \in U,
$$

the strong differential subordination holds; then,

$$
\frac{I R_{\lambda, l}^{m+1} f(z, \zeta)}{I R_{\lambda, l}^{m} f(z, \zeta)} \prec \prec g(z, \zeta), \zeta \in \bar{U}, z \in U,
$$

and this result is sharp.

Proof. We can write $I R_{\lambda, l}^{m} f(z, \zeta)=z+\sum_{j=n+1}^{\infty}\left(\frac{1+\lambda(j-1)+l}{l+1}\right)^{m} C_{m+j-1}^{m} a_{j}^{2}(\zeta) z^{j}, \zeta \in \bar{U}, z \in U$. for $f(z, \zeta)=z+\sum_{j=n+1}^{\infty} a_{j}(\zeta) z^{j} \in \mathcal{A}_{n \zeta}^{*}$.

Consider $p(z, \zeta)=\frac{I R_{\lambda, l}^{m+1} f(z, \zeta)}{I R_{\lambda, l}^{m} f(z, \zeta)}=\frac{z+\sum_{j=n+1}^{\infty}\left(\frac{1+\lambda(j-1)+l}{l+1}\right)^{m+1} C_{m+j}^{m+1} a_{j}^{2}(\zeta) z^{j}}{z+\sum_{j=n+1}^{\infty}\left(\frac{1+\lambda(j-1)+l}{l+1}\right)^{m} C_{m+j-1}^{m} a_{j}^{2}(\zeta) z^{j}}=$ $\frac{1+\sum_{j=n+1}^{\infty}\left(\frac{1+\lambda(j-1)+l}{l+1}\right)^{m+1} C_{m+j}^{m+1} a_{j}^{2}(\zeta) z^{j-1}}{1+\sum_{j=n+1}^{\infty}\left(\frac{1+\lambda(j-1)+l}{l+1}\right)^{m} C_{m+j-1}^{m} a_{j}^{2}(\zeta) z^{j-1}}$.

We have $p_{z}^{\prime}(z, \zeta)=\frac{\left(I R_{\lambda, l}^{m+1} f(z, \zeta)\right)_{z}^{\prime}}{I R_{\lambda, l}^{m} f(z, \zeta)}-p(z, \zeta) \cdot \frac{\left(I R_{\lambda, \lambda}^{m} f(z, \zeta)\right)_{z}^{\prime}}{I R_{\lambda, l}^{m} f(z, \zeta)}$, and $p(z, \zeta)+z p_{z}^{\prime}(z, \zeta)=$ $\left(\frac{z I R_{\lambda, l}^{m+1} f(z, \zeta)}{I R_{\lambda, l}^{m} f(z, \zeta)}\right)_{z}^{\prime}$

Relation (14) can be written as $p(z, \zeta)+z p_{z}^{\prime}(z, \zeta) \prec \prec h(z, \zeta)=g(z, \zeta)+z g_{z}^{\prime}(z, \zeta)$, $\zeta \in \bar{U}, z \in U_{\text {, }}$ and applying Lemma 2 , we obtain $p(z, \zeta) \prec \prec g(z, \zeta), \zeta \in \bar{U}, z \in U$, which is equivalent with $\frac{I R_{\lambda, l}^{m+1} f(z, \zeta)}{I R_{\lambda, l}^{m} f(z, \zeta)} \prec \prec g(z, \zeta), \zeta \in \bar{U}, z \in U$.

Theorem 6. Let $g$ be a convex function in $U \times \bar{U}$ such that $g(0, \zeta)=1$ and $h(z, \zeta)=g(z, \zeta)+$ $\frac{n \lambda(l+1)}{\lambda(l-m+2)-(l+1)} z g_{z}^{\prime}(z, \zeta), m, n \in \mathbb{N}, \lambda, l \geq 0, \zeta \in \bar{U}, z \in U$. If $f \in \mathcal{A}_{n \zeta}^{*}$ and

$$
\begin{gathered}
\frac{1}{z}\left[\frac{(m+1)(l+1)}{\lambda(l-m+2)-(l+1)} I R_{\lambda, l}^{m+1} f(z, \zeta)-\frac{(m-2)(l+1)}{\lambda(l-m+2)-(l+1)} I R_{\lambda, l}^{m} f(z, \zeta)\right]+ \\
\frac{\lambda(l-m+2)-2(l+1)}{\lambda(l-m+2)-(l+1)}-\frac{2(m-1)(l+1)-2 m \lambda}{\lambda(l-m+2)-(l+1)} \int_{0}^{z} \frac{I R_{\lambda, l}^{m} f(t, \zeta)-t}{t^{2}} d t \prec \prec h(z, \zeta),
\end{gathered}
$$

$\zeta \in \bar{U}, z \in U$, the strong differential subordination holds; then,

$$
\left(I R_{\lambda, l}^{m} f(z, \zeta)\right)_{z}^{\prime} \prec \prec g(z, \zeta), \zeta \in \bar{U}, z \in U,
$$

and this result is sharp.

Proof. For $f(z, \zeta)=z+\sum_{j=n+1}^{\infty} a_{j}(\zeta) z^{j} \in \mathcal{A}_{n \zeta}^{*}$ denote $p(z, \zeta)=\left(I R_{\lambda, l}^{m} f(z, \zeta)\right)_{z}^{\prime}=1+\sum_{j=n+1}^{\infty}\left(\frac{1+\lambda(j-1)+l}{l+1}\right)^{m} C_{m+j-1}^{m} j a_{j}^{2}(\zeta) z^{j-1}$.

We have $p(0, \zeta)=1$ and, after a short computation, $p(z, \zeta)+z p_{z}^{\prime}(z, \zeta)=$ $\frac{1}{z}\left(\frac{m+1}{\lambda} I R_{\lambda, l}^{m+1} f(z, \zeta)-\frac{m-2}{\lambda} I R_{\lambda, l}^{m} f(z, \zeta)\right)+\frac{\lambda(m-1)-(l+1)}{\lambda(l+1)} p(z, \zeta)+$ $\left(1-\frac{m-1}{l+1}-\frac{2}{\lambda}\right)-\frac{2(l+1)(m-1)-2 \lambda m}{\lambda(l+1)} \int_{0}^{z} \frac{I R_{\lambda, l}^{m} f(t, \zeta)-t}{t^{2}} d t$.

Therefore, $\frac{\lambda(l+1)}{\lambda(l-m+2)-(l+1)} z p_{z}^{\prime}(z, \zeta)+p(z, \zeta)=$ $\frac{1}{z}\left[\frac{(m+1)(l+1)}{\lambda(l-m+2)-(l+1)} I R_{\lambda, l}^{m+1} f(z, \zeta)-\frac{(m-2)(l+1)}{\lambda(l-m+2)-(l+1)} I R_{\lambda, l}^{m} f(z, \zeta)\right]+\frac{\lambda(l-m+2)-2(l+1)}{\lambda(l-m+2)-(l+1)}-$ 
$\frac{2(l+1)(m-1)-2 \lambda m}{\lambda(l-m+2)-(l+1)} \int_{0}^{z} \frac{I R_{\lambda, l}^{m} f(t, \zeta)-t}{t^{2}} d t$

We obtain $p(z, \zeta)+\frac{\lambda(l+1)}{\lambda(l-m+2)-(l+1)} z p_{z}^{\prime}(z, \zeta) \prec \prec h(z, \zeta)=g(z, \zeta)+\frac{n \lambda(l+1)}{\lambda(l-m+2)-(l+1)} z g_{z}^{\prime}(z, \zeta)$, $z \in U, \zeta \in \bar{U}$. Lemma 2 implies $p(z, \zeta) \prec \prec g(z, \zeta), z \in U, \zeta \in \bar{U}$, which is equivalent with $\left(I R_{\lambda, l}^{m} f(z, \zeta)\right)_{z}^{\prime} \prec \prec g(z, \zeta), z \in U, \zeta \in \bar{U}$, a sharp result.

Theorem 7. Let $h$ be a convex function in $U \times \bar{U}$ with $h(0, \zeta)=1$. If $n, m \in \mathbb{N}, \lambda, l \geq 0$, $f \in \mathcal{A}_{n \zeta}^{*}$ and

$$
\begin{gathered}
\frac{1}{z}\left[\frac{(m+1)(l+1)}{\lambda(l-m+2)-(l+1)} I R_{\lambda, l}^{m+1} f(z, \zeta)-\frac{(m-2)(l+1)}{\lambda(l-m+2)-(l+1)} I R_{\lambda, l}^{m} f(z, \zeta)\right]+ \\
\frac{\lambda(l-m+2)-2(l+1)}{\lambda(l-m+2)-(l+1)}-\frac{2(l+1)(m-1)-2 \lambda m}{\lambda(l-m+2)-(l+1)} \int_{0}^{z} \frac{I R_{\lambda, l}^{m} f(t, \zeta)-t}{t^{2}} d t \prec \prec h(z, \zeta),
\end{gathered}
$$

$\zeta \in \bar{U}, z \in U$, the strong differential subordination holds; then,

$$
\left(I R_{\lambda, l}^{m} f(z, \zeta)\right)_{z}^{\prime} \prec \prec g(z, \zeta) \prec \prec h(z, \zeta), \zeta \in \bar{U}, z \in U,
$$

where the convex best dominant is $g(z, \zeta)=\frac{\lambda(l-m+2)-(l+1)}{n \lambda(l+1) z^{\frac{\lambda(l-m+2)-(l+1)}{n \lambda(l+1)}}} \int_{0}^{z} h(t, \zeta) t^{\frac{\lambda(l-m+2)-(l+1)}{n \lambda(l+1)}-1} d t$.

Proof. For $f(z, \zeta)=z+\sum_{j=n+1}^{\infty} a_{j}(\zeta) z^{j}$, denote $p(z, \zeta)=\left(I R_{\lambda, l}^{m} f(z, \zeta)\right)_{z}^{\prime}$ and $p(0, \zeta)=1$.

Differentiating with respect to $z$, we can write that $p(z, \zeta)+\frac{\lambda(l+1)}{\lambda(l-m+2)-(l+1)} z p_{z}^{\prime}(z, \zeta)=$ $\frac{1}{z}\left[\frac{(m+1)(l+1)}{\lambda(l-m+2)-(l+1)} I R_{\lambda, l}^{m+1} f(z, \zeta)-\frac{(m-2)(l+1)}{\lambda(l-m+2)-(l+1)} I R_{\lambda, l}^{m} f(z, \zeta)\right]+\frac{\lambda(l-m+2)-2(l+1)}{\lambda(l-m+2)-(l+1)}-$ $\frac{2(l+1)(m-1)-2 \lambda m}{\lambda(l-m+2)-(l+1)} \int_{0}^{z} \frac{I R_{\lambda, l}^{m} f(t, \zeta)-t}{t^{2}} d t$ and the strong differential subordination from the hypothesis can be written $p(z, \zeta)+\frac{\lambda(l+1)}{\lambda(l-m+2)-(l+1)} z p^{\prime}(z, \zeta) \prec \prec h(z, \zeta), \zeta \in \bar{U}, z \in U$.

We can apply Lemma 1 for $\gamma=\frac{\lambda(l-m+2)-(l+1)}{\lambda(l+1)}$ since $p(z, \zeta) \in \mathcal{H}^{*}[1, n, \zeta]$ and we obtain $p(z, \zeta) \prec \prec g(z, \zeta) \prec \prec h(z, \zeta), \zeta \in \bar{U}, z \in U$, i.e., $\left(I R_{\lambda, l}^{m} f(z, \zeta)\right)_{z}^{\prime} \prec \prec g(z, \zeta)=$ $\frac{\lambda(l-m+2)-(l+1)}{n \lambda(l+1) z^{\frac{\lambda(l-m+2)-(l+1)}{n \lambda(l+1)}}} \int_{0}^{z} h(t, \zeta) t^{\frac{\lambda(l-m+2)-(l+1)}{n \lambda(l+1)}-1} d t \prec \prec h(z, \zeta), \zeta \in \bar{U}, z \in U$, and $g(z, \zeta)$ is the convex best dominant.

Corollary 2. Let $0 \leq \beta<1$ and the convex function in $U \times \bar{U}, h(z, \zeta)=\frac{\zeta+(2 \beta-\zeta) z}{1+z}$. If $n, m \in \mathbb{N}$, $\lambda, l \geq 0, f \in \mathcal{A}_{n \zeta}^{*}$ and

$$
\begin{gathered}
\frac{1}{z}\left[\frac{(m+1)(l+1)}{\lambda(l-m+2)-(l+1)} I R_{\lambda, l}^{m+1} f(z, \zeta)-\frac{(m-2)(l+1)}{\lambda(l-m+2)-(l+1)} I R_{\lambda, l}^{m} f(z, \zeta)\right]+ \\
\frac{\lambda(l-m+2)-2(l+1)}{\lambda(l-m+2)-(l+1)}-\frac{2(m-1)(l+1)-2 m \lambda}{\lambda(l-m+2)-(l+1)} \int_{0}^{z} \frac{I R_{\lambda, l}^{m} f(t, \zeta)-t}{t^{2}} d t \prec \prec h(z, \zeta),
\end{gathered}
$$

$\zeta \in \bar{U}, z \in U$, the strong differential subordination holds; then,

$$
\left(I R_{\lambda, l}^{m} f(z, \zeta)\right)_{z}^{\prime} \prec \prec g(z, \zeta) \prec \prec h(z, \zeta), \zeta \in \bar{U}, z \in U,
$$

where the convex best dominant $g$ is given by $g(z, \zeta)=2 \beta-\zeta+\frac{2(\zeta-\beta)[\lambda(l-m+2)-(l+1)]}{\lambda(l+1) n z \frac{\lambda(l-m+2)-(l+1)}{\lambda(l+1) n}} \int_{0}^{z} \frac{t^{\frac{\lambda(l-m+2)-(l+1)}{\lambda(l+1) n}-1}}{1+t} d t, \zeta \in \bar{U}, z \in U$. 
Proof. Considering $p(z, \zeta)=\left(I R_{\lambda, l}^{m} f(z, \zeta)\right)_{z}^{\prime}$, the strong differential subordination (17) becomes

$$
p(z, \zeta)+\frac{\lambda(l+1)}{\lambda(l-m+2)-(l+1)} z p_{z}^{\prime}(z, \zeta) \prec \prec h(z, \zeta)=\frac{\zeta+(2 \beta-\zeta) z}{1+z}, \quad \zeta \in \bar{U}, z \in U .
$$

Applying Lemma 1 for $\gamma=\frac{\lambda(l-m+2)-(l+1)}{\lambda(l+1)}$, we obtain $p(z, \zeta) \prec \prec g(z, \zeta) \prec \prec h(z, \zeta)$, $\zeta \in \bar{U}, z \in U$, which is equivalent with

$$
\begin{gathered}
\left(I R_{\lambda, l}^{m} f(z, \zeta)\right)_{z}^{\prime} \prec \prec g(z, \zeta)=\frac{\lambda(l-m+2)-(l+1)}{n \lambda(l+1) z^{\frac{\lambda(l-m+2)-(l+1)}{n \lambda(l+1)}}} \int_{0}^{z} h(t, \zeta) t^{\frac{\lambda(l-m+2)-(l+1)}{n \lambda(l+1)}-1} d t \\
=\frac{\lambda(l-m+2)-(l+1)}{n \lambda(l+1) z^{\frac{\lambda(l-m+2)-(l+1)}{n \lambda(l+1)}}} \int_{0}^{z} t^{\frac{\lambda(l-m+2)-(l+1)}{n \lambda(l+1)}-1} \frac{\zeta+(2 \beta-\zeta) t}{1+t} d t \\
=2 \beta-\zeta+\frac{2(\zeta-\beta)[\lambda(l-m+2)-(l+1)]}{\lambda(l+1) n z^{\frac{\lambda(l-m+2)-(l+1)}{\lambda(l+1) n}}} \int_{0}^{z} \frac{\frac{\lambda}{\frac{\lambda(l-m+2)-(l+1)}{\lambda(l+1) n}-1}_{1+t}}{1+t} d t \quad z \in U, \zeta \in \bar{U} .
\end{gathered}
$$

\section{Discussion}

Generalizing the concept of differential subordination and using the operator defined by using the multiplier transformation and Ruscheweyh operator, further study is carried out and new strong subordinations are obtained, also giving their best dominant. Interesting corollaries are stated using particular functions as the best dominant of the strong subordinations studied in the theorems described in this paper. Using the strong subordination and strong superordination for some differential operators, we can obtain some new properties of the studied operators. The operator can be applied for the introduction of other subclasses of analytic functions and further investigations related to symmetry properties, distortion theorems, coefficient estimates, neighborhoods and the radii of starlikeness, closure theorems, convexity or close-to-convexity of functions from the defined class can be performed.

Funding: This research received no external funding.

Institutional Review Board Statement: Not applicable.

Informed Consent Statement: Not applicable.

Data Availability Statement: Not applicable.

Conflicts of Interest: The author declares no conflict of interest.

\section{References}

1. Alexander, J.W. Functions which map the interior of the unit circle upon simple regions. Ann. Math. 1915, 17, 12-22. [CrossRef]

2. Bernardi, S.D. Convex and starlike univalent functions. Trans. Am. Math. Soc. 1969, 135, 429-446. [CrossRef]

3. Ruscheweyh, S. New criteria for univalent functions. Proc. Am. Math. Soc. 1975, 49, 109-115. [CrossRef]

4. Sălăgean, G.S. Subclasses of Univalent Functions. In Complex Analysis_Fifth Romanian-Finnish Seminar; Lecture Notes in Mathematics; Springer: Berlin, Germany, 1983; Volume 1013, pp. 362-372.

5. Oros, G.I. New differential subordinations obtained by using a differential-integral Ruscheweyh-Libera operator. Miskolc Math. Notes 2020, 21, 303-317. [CrossRef]

6. Acu, M.; Oros, G. Starlikeness condition for a new differential-integral operator. Mathematics 2020, 8, 694. [CrossRef]

7. Pall-Szabo, A.O. On a class of univalent functions defined by Sălăgean integro-differential operator. Miskolc Math. Notes 2018, 19, 1095-1106. [CrossRef]

8. Dziok, J.; Srivastava, H.M. Classes of analytic functions associated with the generalized hypergeometric function. Appl. Math. Comput. 1999, 103, 1-13. [CrossRef] 
9. Srivastava, H.M. Some Fox-Wright generalized hypergeometric functions and associated families of convolution operators. Appl. Anal. Discret. Math. 2007, 1, 56-71.

10. Cătaş, A. Sandwich theorems associated with new multiplier transformations. J. Comput. Anal. Appl. 2011, 13, 663-672.

11. Alb Lupaş, A. Certain strong differential subordinations using a multiplier transformation and Ruscheweyh operator. Int. J. Open Probl. Complex Anal. 2011, 3, 1-8.

12. Bauer, K.W.; Ruscheweyh, S. Differential Operators for Partial Differential Equations and Function Theoretic Applications; Lecture Notes in Mathematics; Springer: Berlin, Germany; New York, NY, USA, 1980; Volume 791.

13. Li, T.; Viglialoro, G. Boundedness for a nonlocal reaction chemotaxis model even in the attraction-dominated regime. Differ. Integral Equ. 2021, 34, 315-336.

14. Li, T.; Pintus, N.; Viglialoro, G. Properties of solutions to porous medium problems with different sources and boundary conditions. Z. Angew. Math. Phys. 2019, 70, 86. [CrossRef]

15. Antonino, J.A.; Romaguera, S. Strong differential subordination to Briot-Bouquet differential equations. J. Differ. Equ. 1994, 114, 101-105. [CrossRef]

16. Oros, G.I.; Oros, G. Strong differential subordination. Turk. J. Math. 2009, 33, 249-257.

17. Oros, G.I. On a new strong differential subordination. Acta Univ. Apulensis 2012, 32, 243-250.

18. Miller, S.S.; Mocanu, P.T. Second order differential inequalities in the complex plane. J. Math. Anal. Appl. 1078, 65, 289-305. [CrossRef]

19. Miller, S.S.; Mocanu, P.T. Differential subordinations and univalent functions. Mich. Math. J. 1981, 28, 157-172. [CrossRef]

20. Miller, S.S.; Mocanu, P.T. Differential Subordinations. Theory and Applications; Marcel Dekker, Inc.: New York, NY, USA; Basel, Switzerland, 2000.

21. Alb Lupaş, A.; Oros, G.I.; Oros, G. On special strong differential subordinations using Sălăgean and Ruscheweyh operators. J. Comput. Anal. Appl. 2012, 14, 266-270.

22. Alb Lupas, A.; Oros, G.I.; Oros, G. A note on special strong differential subordinations using multiplier transformation. J. Comput. Anal. Appl. 2012, 14, 261-265.

23. Alb Lupaş, A. On special strong differential subordinations using a generalized Sălăgean operator and Ruscheweyh derivative. J. Concr. Appl. Math. 2012, 10, 17-23.

24. Alb Lupaş, A. A new comprehensive class of analytic functions defined by multiplier transformation. Math. Comput. Model. 2011, 54, 2355-2362. [CrossRef]

25. Alb Lupaş, A. A note on a certain subclass of analytic functions defined by multiplier transformation. J. Comput. Anal. Appl. 2010, 12, 369-373.

26. Alb Lupaş, A. A note on differential superordinations using a multiplier transformation and Ruscheweyh derivative. Stud. Univ. Babes-Bolyai. Math. 2010, 9, 3-20.

27. Alb Lupaş, A. A note on strong differential subordinations using a generalized Sălăgean operator and Ruscheweyh operator. Commun. Math. Anal. Acta Univ. 2013, 34, 105-114.

28. Alb Lupaş, A. Certain strong differential subordinations using Sălăgean and Ruscheweyh operators. Adv. Appl. Math. Anal. 2011, $6,27-34$. 